\title{
Pragmatisme et activités : des interactions aux transactions
}

Introduction au dossier

Christian Brassac et Manuel Zacklad

\section{CpenEdition}

\section{Journals}

Édition électronique

URL : http://journals.openedition.org/activites/619

DOI : 10.4000 /activites.619

ISSN : 1765-2723

Éditeur

ARPACT - Association Recherches et Pratiques sur les ACTivités

\section{Référence électronique}

Christian Brassac et Manuel Zacklad, «Pragmatisme et activités : des interactions aux transactions », Activités [En ligne], 10-1 | Avril 2013, mis en ligne le 15 avril 2013, consulté le 22 septembre 2020. URL : http://journals.openedition.org/activites/619; DOI : https://doi.org/10.4000/activites.619

\section{(c) (i) (2) $\Theta$}

Activités est mis à disposition selon les termes de la licence Creative Commons Attribution - Pas d'Utilisation Commerciale - Pas de Modification 4.0 International. 


\section{Introduction au dossier \\ Pragmatisme et activités : des interactions aux transactions}

Christian Brassac

Manuel Zacklad

Les recherches en psychologie du travail, en ergonomie, en sciences de la cognition ayant porté, lors de ces vingt dernières années, sur les activités cognitives collaboratives ont été marquées par deux tendances lourdes: (1) la prise en compte accrue du caractère situé de l'activité et de la place des artefacts dans les phénomènes de médiation (objet intermédiaire, artefact cognitif, etc.), (2) la prise en compte du caractère collectif des processus et de l'importance de la dimension informelle de la coopération (interactions conversationnelles, communautés de pratiques, conscience mutuelle, etc.).

La première tendance conduit à des échanges avec l'anthropologie des techniques et la psychologie de l'activité d'inspiration vygotskienne. La seconde avec la sociologie et la psychologie sociale de l'interaction, et plus précisément avec l'analyse conversationnelle des ethnométhodologues et l'interactionnisme symbolique. Malheureusement, force est de constater que ces deux tendances, porteuses d'un renouvellement théorique et méthodologique fécond, dialoguent difficilement : intégration pas vraiment convaincante de la dimension collective de l'activité, prise en compte délicate des transformations des artefacts et des dispositifs organisationnels sur la durée.

L'objectif de ce dossier est de contribuer à un dépassement de ces difficultés en promouvant de nouvelles théories et méthodes d'analyse de l'activité collective s'appuyant sur la pensée pragmatiste; pensée qui connaît depuis plusieurs années un regain d'intérêt à travers l'étude des œuvres de ses fondateurs: Pierce, James, Dewey, Mead. Les approches fondées sur le pragmatisme possèdent en effet plusieurs avantages pour une analyse de l'activité qui combine l'attention aux situations pratiques et l'ouverture sur les significations sociales et politiques de ces dernières: elles permettent de ne pas séparer la pensée et l'action, la gestualité et le langage, le psychisme et l'environnement (psycho)social et matériel...

Le concept de transaction, central dans l'analyse de l'expérience par Dewey ou dans l'étude des conversations par Mead, tout à la fois englobe et prolonge celui d'interaction selon plusieurs directions soulignées récemment par divers auteurs dont les contributeurs à ce dossier: prise en compte intégrée et non cloisonnée des artefacts médiateurs gestuels, langagiers et matériels, attention portée à la fois aux échanges locaux et à leurs généalogies et prolongement dans de multiples séries de situations dérivées, insistance mise sur la transformation simultanée des artefacts et des personnes qui les produisent ou les mobilisent, inscription de toute action dans le cadre d'un échange interpersonnel fût-il institutionnalisé ou intériorisé, etc.

Enfin, dernier avantage majeur, les approches basées sur le pragmatisme appellent de manière résolue à l'interdisciplinarité entre les sciences humaines et sociales tout en les invitant à élargir leur capacité à prendre en compte le rôle de la technique : sociologie, économie, psychologie, philosophie, droit, sciences politiques, sciences de gestion, sciences de la formation, etc. Elles sont en particulier à l'origine de l'institutionnalisme, qui connaît 
également un regain d'intérêt majeur au sein des sciences sociales et particulièrement de l'économie.

$* * * * * * * * * * * * * * * * * * * * * * * * * * *$

C'est dans cet esprit que les auteurs ont construit leur contribution à ce dossier. Leurs textes participent tous, clairement, au «tournant pratique » (practice turn) en tant qu'ils mobilisent efficacement les travaux de deux des pragmatistes historiques, Dewey et Mead. Si cette double référenciation est commune aux quatre contributeurs, ils l'instancient de manières différenciées. Certains proposent de montrer la pertinence de l'idée pragmatiste et la promeuvent en détaillant son opérationnalité pour leurs études empiriques ; d'autres ont pour objectif d'enrichir le corpus des contributions aux débats qui traversent la mobilisation du pragmatisme dans le domaine qui nous intéresse ici; d'autres encore avancent des propositions théoriques incarnées dans des concepts qu'ils ont élaborés et qu'ils mettent en avant. Cet éventail d'approches pourrait faire penser que les textes composant ce dossier ont tous une tonalité uniquement théorique, voire épistémologique. Il n'en est rien. La présence de terrains empiriques constitue un autre point commun aux études présentées ici, qu'ils soient le fait des auteurs eux-mêmes ou qu'ils soient des analyses réalisées par d'autres chercheurs.

Une grande diversité de situations est abordée par les auteurs, de l'orchestre symphonique aux jeux en ligne, du système d'information à un outil de contrôle de gestion, de la téléphonie à l'urgence hospitalière. On trouvera ainsi une histoire décisionnelle s'inscrivant dans un processus stratégique de renouvellement de l'identité artistique d'un orchestre; on lira par ailleurs une étude de cas relative à la mise en œuvre d'un système d'information dans une grande entreprise énergétique ; on s'intéressera à une problématique de conduite de changement dans le milieu universitaire ou encore à des figures de coordinations dans l'activité advenant en milieu hospitalier, dans la sphère numérique ludique ou dans celle du contrôle du trafic téléphonique.

On le voit, le spectre des études de cas mobilisées par les auteurs est large ce qui les amènent à travailler sur des activités cognitives collaboratives très diverses, mais toutes appréhendées sur leurs lieux même d'effectuation. Gageons que cette diversité des démarches théoriques et empiriques, appuyée sur une assise pragmatiste revendiquée, conduira le lecteur à mieux comprendre le sous-titre de ce dossier ; sous-titre suggérant l'idée selon laquelle le recours au pragmatisme, et principalement à deux de ses représentants, permet de théoriser et documenter le dépassement de la notion d'interaction par celle de transaction.

$* * * * * * * * * * * * * * * * * * * * * * * * * * *$

Dans le texte ouvrant ce dossier, dont la revue Organization Studies a aimablement autorisé la traduction, Barbara Simpson se donne un objectif clair. Il s'agit pour elle de contribuer au «tournant pratique » (practice turn), qu'elle dit être 'en progression', dans le domaine des sciences des organisations. C'est la voix de la philosophie pragmatiste, et en particulier celle de George Herbert Mead, son «principal informateur », qu'elle veut faire entendre. En effet cette voix porte, selon elle, à la fois une remise en question de la dominance des théories de l'action rationnelle et de l'action orientée par la norme, et une vision holistique permettant de « réunir les aspects routiniers et créatifs de la pratique tout en transcendant la séparation problématique entre les niveaux individuel et social des analyses » (p. 156). L'exposition de la progression du tournant se poursuit par l'évocation du travail de Mead dont elle extrait deux notions, la transactionnalité et la temporalité, très intriquées dans sa théorie de la socialité. En s'appuyant sur une étude empirique relative à la mise en place d'une stratégie de redéfinition de l'identité artistique d'un orchestre britannique, elle montre en quoi l'approche meadienne de l'action sociale «offre un riche potentiel pour de nouvelles conceptualisations et compréhensions de ce que font effectivement les gens dans les organisations » (p. 166). 
Alexandra Bidet, Manuel Boutet et Frédérique Chave poursuivent le même objectif que la précédente («il en va d'un approfondissement du Practice Turn», p. 173) tout en notant qu'il ne s'agit pas là, simplement, d'une motivation relevant du seul champ théorique, mais aussi d'une nécessité en tant que chercheurs en sociologie du travail - chercheurs dont la préoccupation constante est d'analyser les opérations générant l'action collective d'humains engagés dans des activités coopératives finalisées. Un examen attentif des propositions de George Herbert Mead et John Dewey en la matière les conduit à mobiliser la notion de transaction. Ils l'exploitent de façon très féconde à la fois à partir de l'idée de celui-ci (« la transaction invitant à considérer la primauté du couplage de l'organisme et de l'environnement », p. 176) et de celui-là (la transaction comme étendant l'interaction au-delà de l'interhumain et du seul langagier). Ceci leur permet de rattacher la question de la coopération à celle de l'expérience et de documenter des phénomènes qui touchent à la présence, au cœur des activités collectives, d'artefacts techniques. Trois enquêtes de terrain donnent à voir au lecteur en quoi la notion de transaction amène à considérer des éléments tels que l'autonomie des artefacts techniques, l'activité de tiers ou la distribution temporelle des intérêts comme étant susceptibles de stabiliser des formes de coopération «en dehors d'un horizon d'intelligibilité mutuelle » (p. 177).

La notion de transaction est elle aussi au cœur de la proposition de Manuel Zacklad, qui consiste en un développement de la Sémiotique des Transactions Coopératives qu'il élabore depuis quelques années. Cette théorie concerne au premier chef le travail de management se déployant dans toute entreprise où des humains s'organisent et créent de la valeur. Elle tient pour essentielles les questions liées au cadrage des transactions contribuant à la conduite du changement et à la résistance au changement et puise, dans le corpus des concepts d'origine pragmatiste, les notions de situation et de contexte. Un travail théorique fouillé (sur les vocables action-pratique-activité-transaction ; sur le couple situation/contexte ; sur l'artefact médiateur; etc.) et un examen attentif de l' «orientation ternaire de l'activité » précèdent l'exposé d'une étude de cas relative à l'introduction d'un contrôle gestionnaire renforcé dans l'université française. En appuyant l'essentiel de sa contribution sur une catégorie, la transaction, qui doit beaucoup au pragmatisme, l'auteur dessine une façon personnelle de théoriser le travail d'organisation défini «comme une activité de cadrage des transactions coopératives qui (...) concerne tous les membres de l'entreprise en tant qu'ils s'impliquent dans un travail de management» (p. 192). C'est bien à une approche pragmatiste du management que le lecteur est alors invité à réfléchir.

Philippe Lorino avance quant à lui une proposition théorique qui s'inscrit dans le domaine des recherches sur les organisations et qui s'appuie sur des théories pragmatistes, en particulier celles de l'habitude et de l'enquête. Adoptant une approche actionnelle de l'organisation qui est alors vue comme un processus organisant versus une structure, et qui rend inséparables action et organisation, l'auteur se propose de développer une vision discursive de l'activité collective "centrée sur l'activité elle-même comme discours en actes, plutôt que sur les pratiques et objets langagiers engagés dans ou à propos de l'activité » (p. 222). Il se démarque ainsi des modalités classiques d'analyse de la façon dont sont intimement reliées action collective et émergence continue de significations. Après avoir montré en quoi, historiquement, la notion d'activité a été «oubliée » en sciences des organisations, après avoir brossé à grands traits les tendances en la matière et avoir qualifié l'activité de dialogique et médiatisée, l'auteur suggère de la considérer comme articulant habitudes et enquêtes (au sens pragmatiste) de façon récursive. Il met à l'épreuve cette conceptualisation sur une étude de cas relative à la mise en œuvre d'un système d'information dans une grande entreprise énergétique où des acteurs sont en situation de reconstruire de façon non programmée les cadres discursifs et narratifs de l'activité collective d'achat. 
$\mathrm{Au}$ sortir de l'appréhension des thèses de ces quatre textes promouvant une même ligne thématique, le lecteur pourra sans doute trouver l'empan de ces études trop réduit, le spectre des disciplines trop étroit et/ou la diversité des approches trop limitée. Notre ambition ne tendait aucunement à l'exhaustivité. Elle avait en revanche une dimension «militante » qui peut tenir en une phrase : le renouveau de l'intérêt pour un courant philosophique datant d'un siècle, le pragmatisme américain, ne peut qu'intéresser le lectorat d'une revue dont les tenants épistémologiques, les approches théoriques et les aboutissants méthodologiques et empiriques touchent de si près au champ sémantique du vocable qui en constitue le titre, @ctivités.

\section{REFERENCEMENT}

Brassac, Ch., \& Zacklad, M. (2013). Introduction au dossier «Pragmatisme et activités : des interactions aux transactions ». Activités, 10(1), 151-154, http://www.activites.org/v10n1/v10n1.pdf 